\title{
Association of Side-Branch Treatment and Patient Factors in Left Anterior Descending Artery True Bifurcation Lesions: Analysis from the GRAND-DES Pooled Registry
}

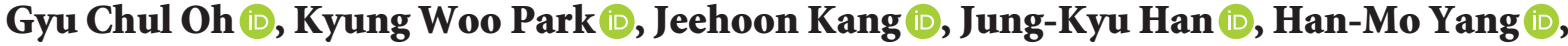 \\ Hyun-Jae Kang $\mathbb{D}$, Bon Kwon Koo $\mathbb{D}$, and Hyo-Soo Kim $\mathbb{D}$ \\ Department of Internal Medicine, Cardiovascular Center, Seoul National University Hospital, Seoul 03080, Republic of Korea \\ Correspondence should be addressed to Kyung Woo Park; kwparkmd@snu.ac.kr
}

Received 14 September 2020; Revised 5 December 2020; Accepted 14 December 2020; Published 28 December 2020

Academic Editor: Joseph Dens

Copyright ( 2020 Gyu Chul Oh et al. This is an open access article distributed under the Creative Commons Attribution License, which permits unrestricted use, distribution, and reproduction in any medium, provided the original work is properly cited.

Background and Objectives. In most bifurcation lesions, keeping the procedure simple by not treating the side-branch (SB) lesion when possible is considered the best method. However, because of improvements with $2^{\text {nd }}$ generation drug-eluting stents (DESs), it remains unknown whether treatment of the SB may improve outcomes in certain subgroups, especially when exclusively using $2^{\text {nd }}$ generation DESs. We report the outcome of SB treatment in a group of patients exclusively receiving newer generation DES for bifurcation PCI. Methods. Patients undergoing PCI to left anterior descending (LAD) bifurcation lesions with contemporary DES were analyzed from a nationwide registry. Baseline risk was assessed using the Age, Creatinine, and Ejection Fraction (ACEF) score. Target lesion failure (TLF), a composite of cardiac death, target vessel myocardial infarction, and target lesion revascularization, was assessed at 3 years. Results. Among 1,089 patients with LAD bifurcation lesions, 548 (50.3\%) patients underwent SB treatment. The SB treatment group showed a nonsignificant, but numerically lower rate of 3-year TLF (6.6\% vs. 9.2\%, HR 0.75, $95 \%$ CI $0.44-1.28, p=0.29)$. In patients with low pretreatment risk $(\mathrm{ACEF}<1.22)$, SB treatment was associated with a lower rate of 3 -year TLF (HR 0.43, 95\%CI 0.19-0.96, $p=0.04$ ), while no significant difference was observed in patients with high risk $(\mathrm{ACEF} \geq 1.22)$. The difference in the low risk group was mostly driven by target lesion revascularization (HR $0.24,95 \% \mathrm{CI}$ $0.08-0.75, p=0.01)$. Conclusions. SB treatment for LAD bifurcation lesions showed favorable long-term outcomes compared with main-branch-only intervention, especially in patients with low pretreatment risk.

\section{Introduction}

Coronary bifurcation lesions are common and account for $15-20 \%$ of all percutaneous coronary interventions (PCI) [1]. Yet, they still pose a significant challenge to many interventional cardiologists. Bifurcation lesions are more complex in anatomy and lead to longer procedure time, requires more contrast volume and resources, and is associated with increased complications and low success rates [2].

Strategies for bifurcation PCI include a 1-stent approach, which is stenting of the main vessel (MV) first with provisional side-branch (SB) stenting, or an elective 2-stent approach. In the era of $1^{\text {st }}$ generation drug-eluting stents (DES), reports from randomized control studies (RCT) and pooled analyses have mostly favored the 1-stent approach. However, there is no concrete evidence behind this recommendation, especially in nonleft main (LM) coronary bifurcations. Furthermore, we do not know whether certain subgroup of patients may actually benefit from SB treatment. The previous studies mostly overlooked the clinical factors that may be important in future outcome of the bifurcation lesion. Analysis of a Korean bifurcation pooled cohort has shown that patients with diabetes were at high risk of adverse events, especially after 2 stenting in bifurcation PCI. Data from the European P2BiTO registry also reported that 
diabetes was associated with increased MACE after bifurcation PCI, regardless of treatment strategy [3].

The age, creatinine, and ejection fraction (ACEF) risk score was first introduced as a simple tool for predicting mortality in elective cardiac operations [4]. The three components in calculating the ACEF score, age, serum creatinine, and ejection fraction, are critical variables that must always be considered when planning and performing PCI. Although initially validated for short-term outcomes, it has also been applied to patients with acute coronary syndrome [5-7] and has been reported to be well correlated with long-term outcomes in all-comer PCI patients [8-10]. The ACEF score reflects the pretreatment risk of the patient and can be easily calculated.

We sought to evaluate the long-term outcomes of left anterior descending (LAD) true bifurcation lesions all treated with second-generation DES and whether the treatment of the SB may be of benefit in certain clinical risk groups, using a nationwide contemporary DES registry.

\section{Materials and Methods}

2.1. Study Design and Patient Population. The study population consists of patients from the Grand-DES registry, the details of which have been published in previous articles [11-13]. A total of 17,286 patients were enrolled at 55 participating centers from 2008 through 2014, and 13,172 patients treated with contemporary DES (5,154 with everolimus-eluting, 3,007 with biolimus-eluting, and 5,011 with zotarolimus-eluting stents) were screened for eligibility. Patients with LM disease and those without true bifurcation lesions were excluded, and 1,089 candidates who underwent PCI for left anterior descending (LAD) coronary true bifurcation lesions were selected for final analysis (Figure 1).

This study was conducted in accordance with the Declaration of Helsinki and has acquired the approval of the ethics committee of each participating center (ClinicalTrials. gov Identifier: NCT03507205). Written consent forms were acquired at the time of enrollment.

2.2. ACEF Score. The ACEF score was calculated using the following formula: $\mathrm{ACEF}=$ age/left ventricular $\mathrm{EF}+1$ (if creatinine $>2.0 \mathrm{mg} / \mathrm{dL}$ ) [4]. Patients with missing creatinine or left ventricular ejection fraction (LVEF) values were excluded in analyses using the ACEF score. Patients were divided into tertiles according to the calculated score, and outcomes were separately assessed in the lower two tertiles (ACEF-LOW; ACEF score <1.22) and highest tertile (ACEFHIGH; ACEF score $\geq 1.22$ ).

2.3. Outcomes and Definitions. The primary outcome was target lesion failure (TLF), defined as a composite of cardiac death, target vessel myocardial infarction (TV-MI), and clinically driven target lesion revascularization (CD-TLR). Secondary outcomes were individual components of the primary outcome, all-cause death, all MI, any revascularization, target vessel revascularization, and stent thrombosis (ST). Individual outcomes were defined according to

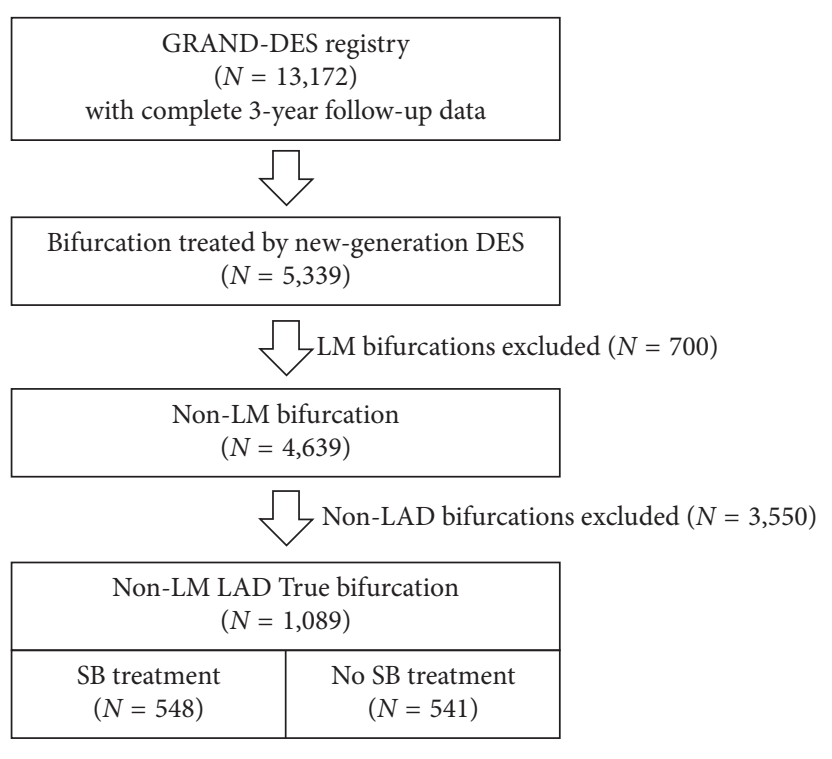

FIGURE 1: Study design. DES, drug-eluting stent; LM, left main coronary artery; LAD, left anterior descending coronary artery; ACEF, age, creatinine, and ejection fraction score; SB, side-branch; Tx, treatment.

Academic Research Consortium definitions [14]. All patients had complete 3-year follow-up assessments, and outcomes were compared according to SB treatment and baseline risk obtained from ACEF scores.

True bifurcation lesions were defined as those having significant $(>50 \%)$ stenosis in both the main branch and the SB (Medina 1, 1, 1; 1, 0, 1; or 0, 1, 1) [15]. Coronary intervention was performed according to current standard guidelines and techniques. Treatment strategy, including the number and type of stent, predilatation, adjunctive balloon inflation, and use of intravascular ultrasound (IVUS), was at the discretion of the operator. Side-branch treatment, unless otherwise specified, was defined as any predilatation, poststenting dilatation, kissing ballooning, or stenting of the SB. Treatment groups were further divided as either ballooning or stenting in subgroup analysis.

2.4. Statistical Analysis. Continuous variables are described as mean \pm standard deviation (SD) and compared using Student's $t$-test or analysis of variance (ANOVA). Categorical variables are shown in numbers and percentages and compared using the chi-square test. Event rates for each outcome were calculated, and comparison of outcomes according to treatment strategy and baseline risk was performed using the log-rank test. Hazard ratios (HR) and 95\% confidence intervals (CI) were calculated using Cox proportional hazard $(\mathrm{PH})$ regression analysis. A multivariate Cox PH model was constructed to adjust for differences in baseline characteristics. The covariates used for multivariate Cox $\mathrm{PH}$ regression analysis are as follows: sex, 3-vessel disease, severe lesion calcification or tortuosity, and ACEF score. All $p$ values were 2 sided, and $p<0.05$ was considered to be significant. All analyses were performed using SPSS version 25 (IBM Crop., Armonk, NY, USA). 


\section{Results and Discussion}

3.1. Baseline Characteristics. The baseline characteristics of the entire study population and those of the SB-treated and nontreated groups are described in Table 1. From a total of 1,089 patients, $548(50.3 \%)$ underwent SB treatment. Patients in the SB-treated group were younger $(63.9 \pm 11.1 \mathrm{vs}$. $65.5 \pm 10.6$ years, $p=0.01)$ and had a lower prevalence of comorbidities and LV dysfunction. There were a higher proportion of patients with single LAD disease in the SBtreated group ( $40.5 \%$ vs. $26.2 \%, p<0.01)$ and more calcified and tortuous lesions in the nontreated group. Among patients undergoing SB treatment, 373 (68.1\%) underwent balloon angioplasty for the SB, while 175 (31.9\%) performed SB stenting. Baseline characteristics for patients according to the type of SB treatment methods are shown in Supplementary Table S1.

The ACEF score was calculated for 904 patients with available creatinine and LVEF values at baseline. The score ranged from 0.49 to 5.36 (mean $\pm \mathrm{SD} ; 1.21 \pm 0.48$ ), with the SB nontreatment group showing a higher mean score compared to the treatment group ( $1.27 \pm 0.54$ vs. $1.15 \pm 0.40$, $p<0.01)$. As previously described, the ACEF score was used to group patients into low (ACEF-LOW) and high (ACEFHIGH) pretreatment risk groups, with 1.22 as the cutoff value. The baseline characteristics of patients in the ACEFLOW and ACEF-HIGH group are shown in Table 2 and Supplementary Table S2.

3.2. Clinical Outcomes. The complete results of the 3-year composite and individual clinical outcomes according to SB treatment and ACEF risk is presented in Table 3. In the whole study population, the rate of TLF was numerically lower in the SB treatment group (6.6 vs. 9.2\%), although the difference was not statistically significant $(\log$-rank $p=0.12)$ (Supplementary Figure S1). Significantly lower rates of cardiac death were observed in the SB treatment group in the unadjusted analysis (log-rank $p=0.02$ ) (Supplementary Figure S2), but the difference was not statistically significant after adjustment for covariates (HR 0.59, 95\% CI 0.27-1.28, $p=0.18$ ).

The ACEF risk score was used to adjust for significant differences in baseline characteristics. The ACEF score correlated with observed outcomes, as ACEF-HIGH patients showed a significantly higher rate of TLF (Figure 2(a)). Although there was no difference in outcomes according to SB treatment in the ACEF-HIGH group, a significant reduction in 3-year TLF was observed with SB treatment in the ACEF-LOW group (Figure 2(b)). The rates of the individual outcomes were also numerically lower in the SB treatment group, with a significant lower rate of CD-TLR observed with SB treatment in the ACEF-LOW group (HR 0.24, 95\% CI $0.08-0.75, p=0.01$ ). Individual outcomes for the ACEFLOW group are presented in Figure 3. Clinical outcomes for the ACEF-HIGH group are presented in Supplementary Table S3.
Rates of stent thrombosis were low throughout the study population, with $0.5 \% / 3 \mathrm{yr}$ for the SB-treated group and $0.6 \% / 3 \mathrm{yr}$ for the nontreated group (Supplementary Figure S3). There was no significant difference according to SB treatment in both the low- and high-risk groups.

3.3. Type ofSB Treatment. Side-branch treatment was further divided into either 2 stenting or SB ballooning. In the whole study population, the rate of TLF was numerically lower both in the 2-stent and SB-ballooning group compared with the nontreatment group, but without statistical significance (HR $0.77,95 \%$ CI $0.42-1.41, p=0.40$ for SB ballooning vs. no treatment; HR 0.81, 95\% CI 0.37-1.74, $p=0.58$, SB stenting vs. no treatment). In the ACEF-LOW group, ballooning of the SB was associated with significantly lower rates of TLF compared with no treatment, but there was no significant difference between stenting and no treatment (Figure 4).

Independent predictors for TLF were assessed using multivariate Cox regression models. After adjusting for covariates, the predictors of poor outcome were increased ACEF score (HR 2.46, 95\% CI 1.92-3.16, $p<0.01$ for every 1point increase) and severe lesion calcification (HR 1.43, 95\% CI 1.51-3.90, $p<0.01$ ) (Supplementary Table S4). In a post hoc subgroup analysis, there was no significant interaction for the primary outcome, except for ACEF risk and sex (Figure 5).

\section{Discussion}

In this analysis reporting the long-term outcomes of LAD bifurcation lesions using a prospective, nationwide registry, we observed that SB treatment was associated with a numerically lower rate of TLF compared with nontreatment. When stratified according to clinical risk, for those with low pretreatment risk according to the ACEF score, SB treatment significantly reduced 3-year TLF, mainly driven by the lower rate of revascularizations. Our analyses suggest that selected lower clinical risk patients may benefit from SB treatment and reduce the need for future revascularization and invasive procedures.

Numerous studies have reported that the 1-stent strategy, also known as the provisional SB stenting strategy, was associated with lower risk of adverse events. Thus, the current consensus for bifurcation PCI is to initially perform main vessel stenting, with provisional stenting of the SB [16]. Provisional stenting remains as an alternative during the procedure and is advised when flow is reduced and angiographic results are suboptimal. Elective 2 stenting is recommended in certain situations such as complex lesions with calcified SB, SB ostial lesions $>5 \mathrm{~mm}$ from the carina, and in bifurcations with major SB $[17,18]$. However, these recommendations mainly focus on angiographic characteristics and tend to overlook the clinical factors associated with the patient. We showed that the potential benefit of SB intervention may be slightly different according to the baseline clinical risk. The ACEF score, a composite of age, 
TABLE 1: Baseline characteristics according to SB treatment.

\begin{tabular}{|c|c|c|c|c|}
\hline & Total $(n=1,089)$ & SB treatment $(n=548)$ & No SB treatment $(n=541)$ & $p$ value \\
\hline \multicolumn{5}{|l|}{ Patient factors } \\
\hline Sex (male, \%) & $762(70.0)$ & $396(72.3)$ & $366(67.7)$ & 0.11 \\
\hline Age (years) & $64.7 \pm 10.9$ & $63.9 \pm 11.1$ & $65.5 \pm 10.6$ & 0.01 \\
\hline BMI $\left(\mathrm{kg} / \mathrm{m}^{2}\right)$ & $24.4 \pm 3.1$ & $24.4 \pm 3.1$ & $24.4 \pm 3.1$ & 0.88 \\
\hline Current smoker & $301(27.6)$ & $157(28.7)$ & $144(26.6)$ & 0.50 \\
\hline \multicolumn{5}{|l|}{ Comorbidities } \\
\hline Hypertension & $669(61.4)$ & $318(58.0)$ & $351(64.9)$ & 0.02 \\
\hline Diabetes & $391(35.9)$ & $173(31.6)$ & $218(40.3)$ & $<0.01$ \\
\hline Dyslipidemia & $668(61.3)$ & $333(60.8)$ & $335(61.9)$ & 0.74 \\
\hline $\mathrm{CKD}$ & $48(4.4)$ & $17(3.1)$ & $31(5.7)$ & 0.04 \\
\hline CVA & $91(8.4)$ & $46(8.4)$ & $45(8.3)$ & 1.00 \\
\hline $\mathrm{PAD}$ & $15(1.4)$ & $8(1.5)$ & $7(1.3)$ & 1.00 \\
\hline $\mathrm{FHx}$ of $\mathrm{CHD}$ & $86(7.9)$ & $47(8.6)$ & $39(7.2)$ & 0.47 \\
\hline Previous MI & $48(4.4)$ & $24(4.4)$ & $24(4.4)$ & 1.00 \\
\hline Present with MI & $306(28.1)$ & $162(29.6)$ & $144(26.6)$ & 0.31 \\
\hline ACS & $647(59.4)$ & $320(58.4)$ & $327(60.4)$ & 0.53 \\
\hline LV dysfunction $(\mathrm{EF}<40 \%)$ & $61(6.6)$ & $23(4.8)$ & $38(8.6)$ & 0.03 \\
\hline Serum $\mathrm{Cr}, \mathrm{mg} / \mathrm{dL}$ & $0.9(0.8-1.1)$ & $0.9(0.8-1.1)$ & $0.9(0.8-1.2)$ & 0.07 \\
\hline History of PCI or CABG & $109(10.0)$ & $52(9.5)$ & $57(10.5)$ & 0.64 \\
\hline \multicolumn{5}{|l|}{ Lesion characteristics } \\
\hline $3 \mathrm{VD}$ & $333(30.6)$ & $139(25.4)$ & $194(35.9)$ & $<0.01$ \\
\hline 2VD & $392(36.0)$ & $187(34.1)$ & $205(37.9)$ & \\
\hline $1 \mathrm{VD}$ & $364(33.4)$ & $222(40.5)$ & $142(26.2)$ & \\
\hline Medina class & & & & 0.96 \\
\hline $1,1,1$ & $798(73.3)$ & $401(73.2)$ & $397(73.4)$ & \\
\hline $1,0,1$ & $89(8.2)$ & $46(8.4)$ & $43(8.0)$ & \\
\hline $0,1,1$ & $202(18.5)$ & $101(18.4)$ & $101(18.7)$ & \\
\hline Calcified lesion & $161(14.8)$ & $57(10.4)$ & $104(19.2)$ & $<0.01$ \\
\hline Tortuous lesion & $226(20.8)$ & $90(16.4)$ & $136(25.1)$ & $<0.01$ \\
\hline \multicolumn{5}{|l|}{ Procedure characteristics } \\
\hline SB balloon angioplasty & $373(34.3)$ & $373(68.1)$ & $0(0)$ & \\
\hline SB stenting & $175(16.1)$ & $175(31.9)$ & $0(0)$ & \\
\hline IVUS-guidance & $471(43.3)$ & $250(45.6)$ & $221(40.9)$ & 0.03 \\
\hline GP IIb-IIIa inhibitor & $48(4.4)$ & $23(4.2)$ & $25(4.6)$ & 0.13 \\
\hline \multicolumn{5}{|l|}{ Medications } \\
\hline DAPT $>1$ year & $575(52.8)$ & $281(51.3)$ & $294(54.3)$ & 0.34 \\
\hline RAS inhibitor & $710(65.2)$ & $356(65.0)$ & $354(65.4)$ & 0.92 \\
\hline Beta-blocker & $710(65.2)$ & $361(65.9)$ & $349(64.5)$ & 0.68 \\
\hline$A C E F$ score $^{*}$ & $1.21 \pm 0.48$ & $1.15 \pm 0.40$ & $1.27 \pm 0.54$ & $<0.01$ \\
\hline
\end{tabular}

Values are mean $\pm \mathrm{SD}$, median (interquartile ranges, $25^{\text {th }}-75^{\text {th }}$ ), or $n(\%)$ (per-patient analysis). ${ }^{*}$ Calculated in 904 patients with available values for creatinine and ejection fraction. SB, side-branch; BMI, body mass index; CVA, cerebrovascular accident; PAD, peripheral artery disease; FHx, family history; CHD, coronary heart disease; MI, myocardial infarction; ACS, acute coronary syndrome; LV, left ventricle; EF, ejection fraction; Cr, creatinine; PCI, percutaneous coronary intervention; CABG, coronary artery bypass graft surgery; 3VD, three-vessel disease; IVUS, intravascular ultrasound; GP, glycoprotein; DAPT, dual antiplatelet therapy; RAS, renin-angiotensin system; ACEF, age, creatinine, and ejection fraction.

TABLE 2: Baseline characteristics according to SB treatment in the low-risk group.

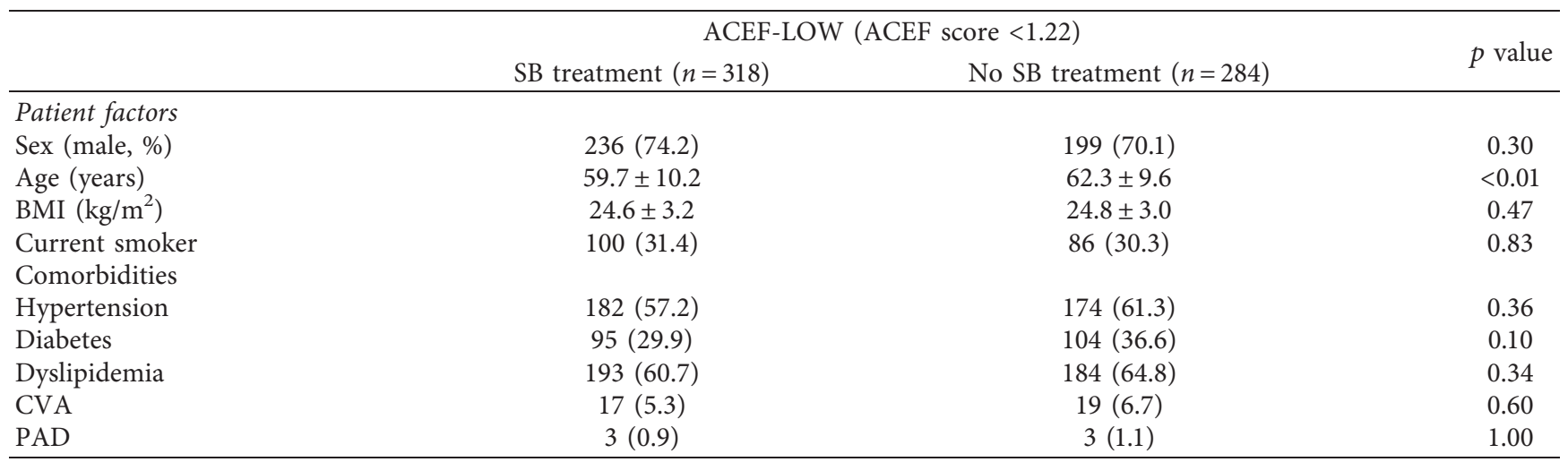


TABLE 2: Continued.

\begin{tabular}{|c|c|c|c|}
\hline & ACEF- & core $<1.22$ ) & م \\
\hline & SB treatment $(n=318)$ & No SB treatment $(n=284)$ & $p$ value \\
\hline FHx of CHD & $32(10.1)$ & $21(7.4)$ & 0.31 \\
\hline Previous MI & $13(4.1)$ & $9(3.2)$ & 0.70 \\
\hline Presentation with MI & $70(22.0)$ & $52(18.3)$ & 0.31 \\
\hline ACS & $165(51.9)$ & $150(52.8)$ & 0.88 \\
\hline LV dysfunction $(\mathrm{EF}<40 \%)$ & $2(0.6)$ & $1(0.4)$ & 1.00 \\
\hline Serum creatinine $(\mathrm{mg} / \mathrm{dL})$ & $0.9(0.8-1.1)$ & $0.9(0.8-1.1)$ & 0.90 \\
\hline History of PCI or CABG & $22(6.9)$ & $20(7.0)$ & 1.00 \\
\hline Lesion characteristics & & & \\
\hline $3 \mathrm{VD}$ & $61(19.2)$ & $93(32.7)$ & $<0.01$ \\
\hline Medina class & & & 0.66 \\
\hline $1,1,1$ & $230(72.3)$ & $213(75.0)$ & \\
\hline $1,0,1$ & $25(7.9)$ & $23(8.1)$ & \\
\hline $0,1,1$ & $63(19.8)$ & $48(16.9)$ & \\
\hline Calcified lesion & $30(9.4)$ & $47(16.5)$ & 0.01 \\
\hline Tortuous lesion & $45(14.2)$ & $76(26.8)$ & $<0.01$ \\
\hline Procedure characteristics & & & \\
\hline SB balloon angioplasty & $213(67.0)$ & $0(0)$ & \\
\hline SB stenting & $105(33.0)$ & $0(0)$ & \\
\hline IVUS-guidance & $159(50.0)$ & $126(44.4)$ & 0.19 \\
\hline GP IIb-IIIa inhibitor & $11(3.5)$ & $6(2.1)$ & 0.45 \\
\hline Medications & & & \\
\hline RAS inhibitor & $204(64.2)$ & $179(63.0)$ & 0.84 \\
\hline Beta-blocker & $209(65.7)$ & $178(62.7)$ & 0.49 \\
\hline ACEF score & $0.94 \pm 0.15$ & $0.99 \pm 0.14$ & $<0.01$ \\
\hline
\end{tabular}

Values are mean \pm SD, median (interquartile ranges, $25^{\text {th }}-75^{\text {th }}$ ), or $n$ (\%) (per-patient analysis). SB, side branch; BMI, body mass index; CVA, cerebrovascular accident; PAD, peripheral artery disease; FHx, family history; CHD, coronary heart disease; MI, myocardial infarction; ACS, acute coronary syndrome; LV, left ventricle; EF, ejection fraction; PCI, percutaneous coronary intervention; CABG, coronary artery bypass graft surgery; 3VD, three-vessel disease; IVUS, intravascular ultrasound; GP, glycoprotein; RAS, renin-angiotensin system; ACEF, age, creatinine, and ejection fraction.

TABLE 3: Three-year composite and individual outcomes according to SB treatment.

\begin{tabular}{|c|c|c|c|c|c|c|c|c|}
\hline \multirow[b]{3}{*}{ Outcomes (n, \%) } & \multicolumn{2}{|c|}{ Whole study population } & \multicolumn{6}{|c|}{ ACEF-LOW group } \\
\hline & \multirow{2}{*}{$\begin{array}{l}\text { SB treatment } \\
\quad(N=548)\end{array}$} & \multirow{2}{*}{$\begin{array}{c}\text { No SB } \\
\text { treatment } \\
(N=541)\end{array}$} & \multicolumn{2}{|c|}{ Adjusted $\mathrm{HR} \dagger$} & \multirow{2}{*}{$\begin{array}{l}\text { SB treatment } \\
\qquad(N=318)\end{array}$} & \multirow{2}{*}{$\begin{array}{c}\text { No SB } \\
\text { treatment } \\
(N=284)\end{array}$} & \multicolumn{2}{|c|}{ Adjusted HR $\dagger$} \\
\hline & & & HR (95\% CI) & $\begin{array}{c}p \\
\text { value }\end{array}$ & & & HR (95\% CI) & $\begin{array}{c}p \\
\text { value }\end{array}$ \\
\hline Target lesion failure* & $36(6.6)$ & $50(9.2)$ & $\begin{array}{c}0.75 \\
(0.44-1.28)\end{array}$ & 0.29 & $9(2.8)$ & $21(7.4)$ & $\begin{array}{c}0.43 \\
(0.19-0.96)\end{array}$ & 0.04 \\
\hline Cardiac death & $16(2.9)$ & $31(5.7)$ & $\begin{array}{c}0.59 \\
(0.27-1.28)\end{array}$ & 0.18 & $3(0.9)$ & $7(2.5)$ & $\begin{array}{c}0.57 \\
(0.14-2.32)\end{array}$ & 0.43 \\
\hline Target vessel MI & $3(0.5)$ & $5(0.9)$ & $\begin{array}{c}0.42 \\
(0.07-2.42)\end{array}$ & 0.33 & $2(0.6)$ & $4(1.4)$ & $\begin{array}{c}0.64 \\
(0.11-3.75)\end{array}$ & 0.62 \\
\hline CD-TLR & $17(3.1)$ & $20(3.7)$ & $\begin{array}{c}0.75 \\
(0.35-1.62)\end{array}$ & 0.46 & $4(1.3)$ & $15(5.3)$ & $\begin{array}{c}0.24 \\
(0.08-0.75)\end{array}$ & 0.01 \\
\hline Definite/probable ST & $3(0.5)$ & $3(0.6)$ & $\begin{array}{c}0.89 \\
(0.07-11.83)\end{array}$ & 0.93 & 0 & $1(0.4)$ & - & - \\
\hline All-cause death & $24(4.4)$ & $47(8.7)$ & $\begin{array}{c}0.69 \\
(0.38-1.27)\end{array}$ & 0.24 & $5(1.6)$ & $8(2.8)$ & $\begin{array}{c}0.97 \\
(0.30-3.08)\end{array}$ & 0.95 \\
\hline Any MI & $6(1.1)$ & $7(1.3)$ & $\begin{array}{c}0.78 \\
(0.23-2.65)\end{array}$ & 0.70 & $4(1.3)$ & $5(1.8)$ & $\begin{array}{c}0.89 \\
(0.23-3.47)\end{array}$ & 0.87 \\
\hline $\begin{array}{l}\text { Any repeat } \\
\text { revascularization }\end{array}$ & $56(10.2)$ & $50(9.2)$ & $\begin{array}{c}1.12 \\
(0.72-1.74)\end{array}$ & 0.62 & $25(7.9)$ & $29(10.2)$ & $\begin{array}{c}0.82 \\
(0.47-1.44)\end{array}$ & 0.49 \\
\hline $\begin{array}{l}\text { Target vessel } \\
\text { revascularization }\end{array}$ & $28(5.1)$ & $30(5.5)$ & $\begin{array}{c}0.96 \\
(0.52-1.78)\end{array}$ & 0.90 & $10(3.1)$ & $20(7.0)$ & $\begin{array}{c}0.48 \\
(0.22-1.05)\end{array}$ & 0.07 \\
\hline
\end{tabular}

${ }^{*}$ Composite of cardiac death, target vessel MI, and CD-TLR. †Adjusted for sex, severe lesion calcification, tortuous lesion, 3-vessel disease, and ACEF score. SB, side branch; HR, hazard ratio; MI, myocardial infarction; CD-TLR, clinically driven target lesion revascularization; ST, stent thrombosis. 


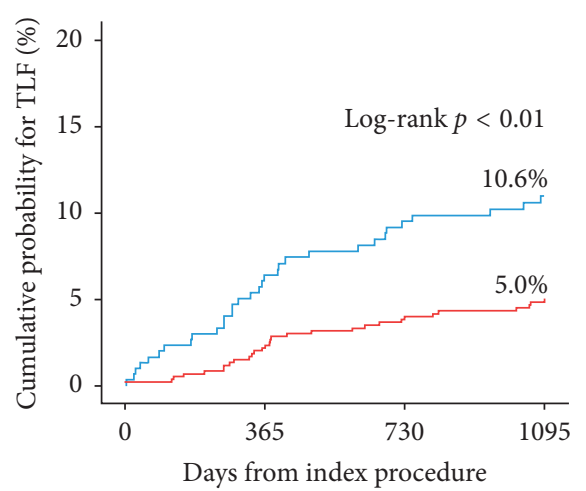

Number at risk

$\begin{array}{ccccr}\text { ACEF-HIGH } & 302 & 273 & 260 & 217 \\ \text { ACEF-LOW } & 602 & 586 & 576 & 466 \\ & & & \\ & & \text { ACEF-HIGH (ACEF score } \geq 1.22 \text { ) } \\ & & \text { ACEF-LOW (ACEF score }<1.22 \text { ) }\end{array}$

(a)

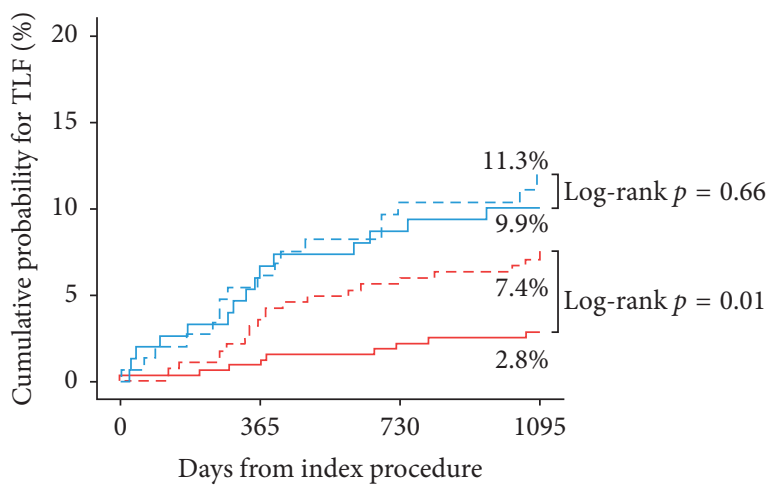

Number at risk (ACEF-LOW)

$\begin{array}{lllll}\text { SB treat }(-) & 284 & 273 & 267 & 215 \\ \text { SB treat }(+) & 318 & 313 & 309 & 251\end{array}$

— ACEF-HIGH (ACEF score $\geq 1.22$ )

- ACEF-LOW (ACEF score $<1.22$ )

- - - No SB treatment

— SB treatment

(b)

FIGURE 2: Kaplan-Meier curves for 3-year TLF according to the (a) ACEF risk group and (b) side-branch treatment. TLF, target lesion failure; ACEF, age, creatinine, and ejection fraction score; and SB, side branch.
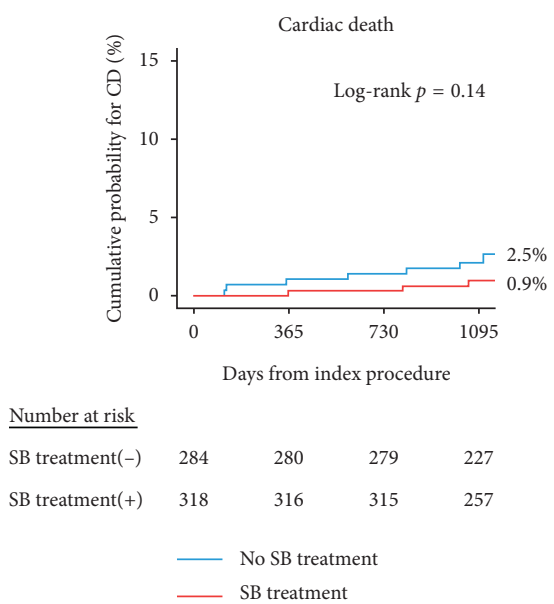
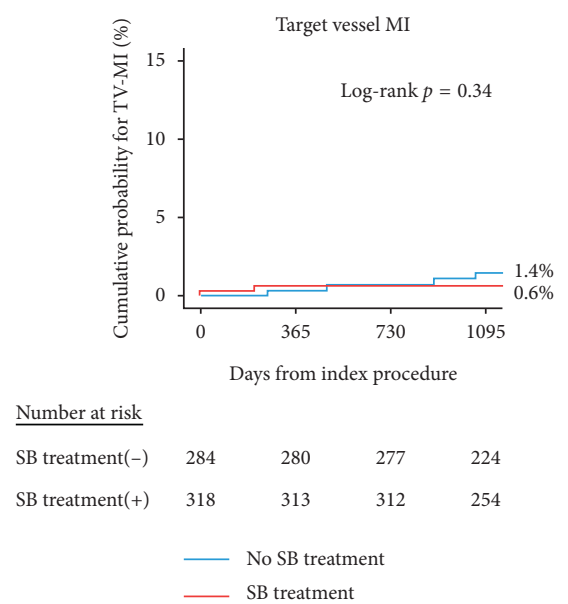

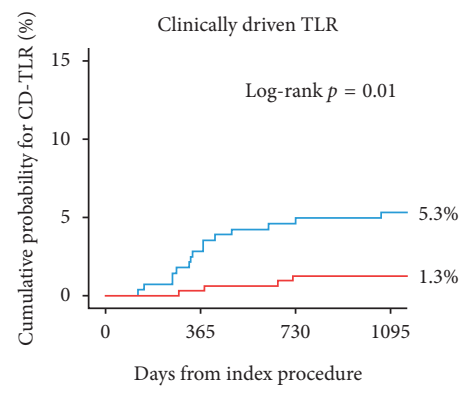

$\underline{\text { Number at risk }}$

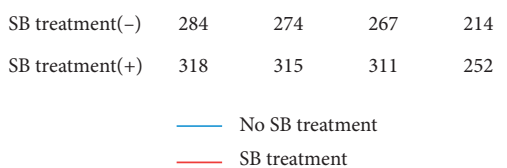

FIgURE 3: Kaplan-Meier curve for 3-year individual outcomes according to side-branch treatment in the ACEF-LOW group. MI, myocardial infarction; TLR, target lesion revascularization; and SB, side branch; ACEF, age, creatinine, and ejection fraction score.

renal, and LV systolic function, was correlated with outcome and the benefit of SB intervention in bifurcation PCI.

Interestingly, TLF rates for patients in the high ACEF risk group were not affected by SB treatment, but there was a significant difference for the low-risk group. The results of individual outcomes show that this difference was mostly driven by CD-TLR, occurring mostly in the 6- to 18 -month period, suggesting that proper full treatment of the SB at the time of the initial procedure might help in preventing future interventions.

The patients analyzed in the present study were exclusively those with newer generation DES. While the bulk of the data comparing one vs. two-stent strategy in bifurcation
PCI was from the 1st generation DES era, newer generation DES have been reported to improve outcomes [19], and in studies comparing 2 stenting between 1 st and 2nd generation DES, newer DES showed significantly superior outcomes to the older generation DES [20]. Development of newer DES with thinner struts and improved deliverability has allowed for lower restenosis and higher success rates, lessening the gap between SB treatment strategies. In our analysis, 3-year TLF was numerically lower in the 2-stent group. Furthermore, this trend was consistent regardless of the individual component of TLF. Similarly, in an all-comers randomized trial evaluating the effect of final kissing ballooning and 2 stenting using newer DES, 1-year 


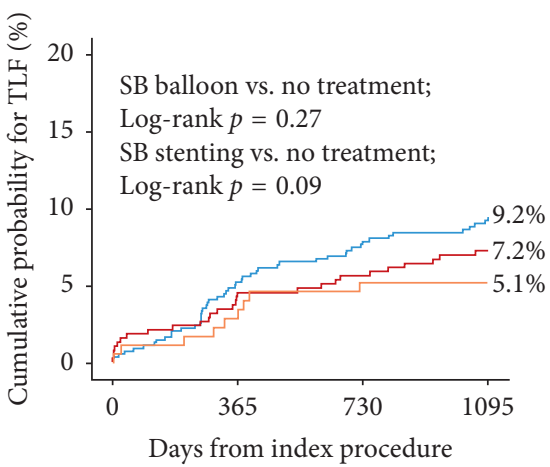

Number at risk

\begin{tabular}{lcccc} 
SB treatment(-) 541 & 504 & 488 & 394 \\
SB ballooning & 373 & 354 & 350 & 279 \\
SB stenting & 175 & 167 & 162 & 140 \\
& & \multicolumn{4}{l}{ No SB treatment } \\
& - SB ballooning \\
& & SB stenting
\end{tabular}

(a)

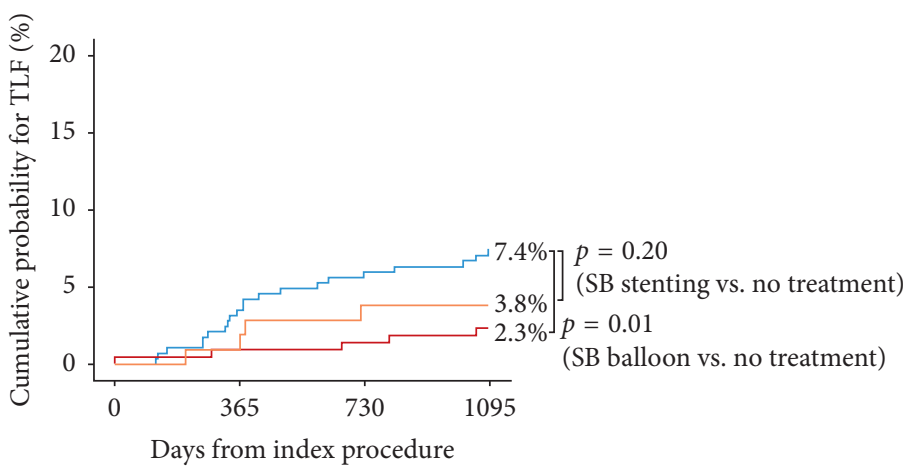

Number at risk

$\begin{array}{lrrrr}\text { SB treatment(-) } 284 & 273 & 267 & 215 \\ \text { SB ballooning } & 213 & 210 & 209 & 164 \\ \text { SB stenting } & 105 & 103 & 100 & 87\end{array}$

(b)

FigURE 4: Kaplan-Meier curves for 3-year target lesion failure according to side-branch treatment method. (a) Whole study population. (b) ACEF-LOW group. SB, side branch. Predictors of composite outcome and post hoc analysis.

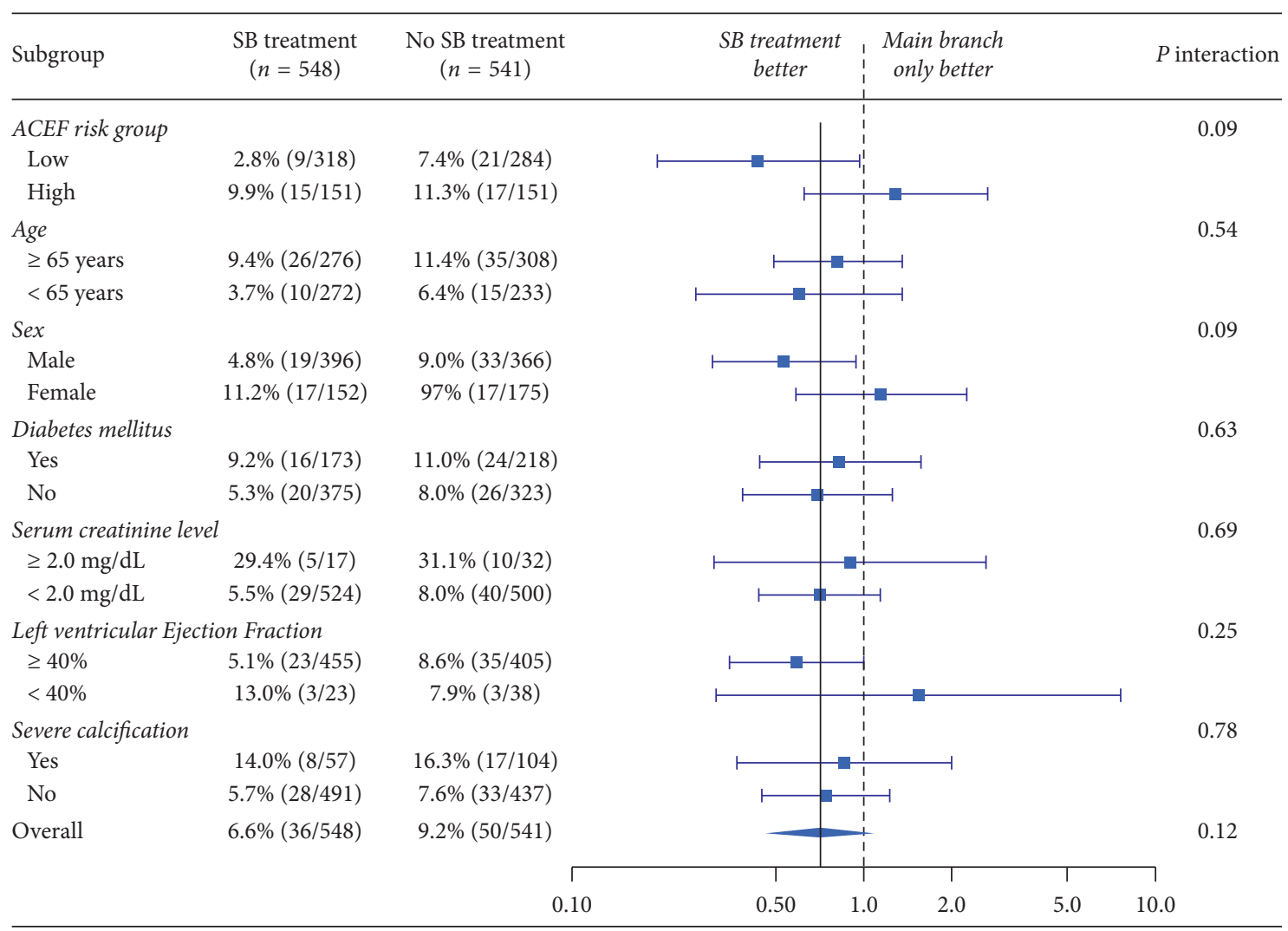

FIGURE 5: Subgroup analysis according to patient factors. SB, side branch; ACEF, age, creatinine, and ejection fraction score. 
angiographic and clinical outcomes were excellent in all groups once the procedure was successfully performed [21].

For patients with SB treatment, $16.1 \%$ underwent PCI with 2 stents, and $34.3 \%$ underwent balloon angioplasty of the SB with or without final kissing ballooning. No significant difference in outcomes was observed according to the type of SB treatment. For patients with low ACEF score, both balloon angioplasty and SB stenting were associated with a reduction in TLF compared with the non-SB treatment group. Balloon angioplasty of the SB, preferably followed by final kissing ballooning (FKB), could be an adequate option for patients with low pretreatment risk.

Outcomes of bifurcation PCI may rely on various patient, lesion characteristics, device selection, and operatorrelated factors. Intravascular ultrasound (IVUS) and optical coherence tomography (OCT) can visualize and calculate the complex anatomy of a bifurcation lesion, making it possible to predict SB compromise beforehand [22, 23]. Techniques such as proximal optimization (POT) and final kissing ballooning, jailed wire, and balloon methods have also allowed for better results in bifurcation PCI. Furthermore, physiologic assessment of the SB using fractional flow reserve (FFR) has also helped select which SB to treat [24].

The ACEF score is a simple, intuitive method that can be calculated in a matter of seconds. Simple and easily available clinical variables may help us guide our procedures. It has also been shown that the addition of clinical variables to the SYNTAX score, the so-called SYNTAX score II, improved the risk stratification capability of the score suggesting the importance of incorporating simple clinical variables into the decision-making process [25].

In the era of evidence-based medicine, adhering to treatment guidelines or expert opinions is important. However, for non-LM coronary bifurcation lesions, the evidence is scarce and sometimes contradictory. In line with current recommendations, most interventionists will probably leave the side-branch alone without definite flow compromise. The results of the current analysis suggest that patient factors such as age and renal function are powerful prognostic factors, and those with high pretreatment risk have unfavorable outcomes. However, complete revascularization might be a better option for those with low pretreatment risk, as they are more likely to receive additional revascularization procedures in the near future and lead to an extended period of antiplatelet therapy. Of course, one has to always keep in mind that routine practice of SB treatment can lead to longer procedure time, more wires, balloons, increased use of contrast, and radiation exposure. The interventionist should carefully anticipate the risk of SB compromise and know how to select the patient who will benefit from elective SB treatment.

\section{Limitations}

There are several limitations to the current study. First of all, the patient population was not derived from a bifurcationdedicated registry and was a subgroup of a pooled DES registry. Criteria on whether to treat the side branch was not predefined, and the decision to treat a certain side branch relied heavily on the operator, which could have influenced outcomes. Second, there were significant differences in baseline characteristics between groups. Patients whose side branches were treated were generally younger, had less comorbidities, and were more likely not to have 3 vessel disease. Although a multivariate Cox Proportional Hazards model was used to adjust for differences, the results need to be interpreted with caution. Third, we were not able to assess whether SB treatment was a result of bail-out treatment (initial one-stent strategy but ended up being a two-stent procedure) or if it was a planned two-stent approach. Finally, there were no data on whether final POT was performed, a factor which is known to be associated with outcomes in bifurcation PCI.

\section{Conclusions}

For patients undergoing PCI of LAD bifurcation lesions, lower clinical risk patients may potentially benefit from complete treatment of the SB by reducing the need for future repeat revascularization.

\section{Data Availability}

The clinical and procedural data used to support the findings of this study may be released upon request and approval by the institutional review board of Seoul National University Hospital. Requests should be made to the corresponding author.

\section{Conflicts of Interest}

The authors declare that there are no conflicts of interest regarding the publication of this paper.

\section{Acknowledgments}

The authors thank the study collaborators for data recruitment and follow-up. This study was supported by grants from the Seoul National University Hospital (Grant nos. 062011-2830 and 06-2010-1090).

\section{Supplementary Materials}

Supplementary Table S1. Baseline characteristics according to the side-branch treatment method. Supplementary Table S2. Patient characteristics according to ACEF risk. Supplementary Table S3. Three-year composite and individual outcomes according to SB treatment in the ACEF-HIGH group. Supplementary Table S4. Factors associated with TLF according to the multivariate Cox regression model. Supplementary Figure S1. Kaplan-Meier curve for 3-year TLF according to side-branch treatment. Supplementary Figure S2. Kaplan-Meier curve for 3-year individual outcomes according to side-branch treatment. Supplementary Figure S3. Kaplan-Meier curve for 3-year definite/probable stent thrombosis according to side-branch treatment. (Supplementary Materials) 


\section{References}

[1] T. K. Steigen, M. Maeng, R. Wiseth et al., "Randomized study on simple versus complex stenting of coronary artery bifurcation lesions," Circulation, vol. 114, no. 18, pp. 1955-1961, 2006.

[2] A. Erglis and A. Colombo, "Bifurcation disease," JACC: Cardiovascular Interventions, vol. 1, no. 3, pp. 218-226, 2008.

[3] L. Di Serafino, H. Gamra, P. Cirillo et al., "P1962Impact of Dual Antiplatelet Therapy duration on clinical outcome after stent implantation for coronary bifurcation lesions: results from the Euro Bifurcation Club - P2BiTO - registry," European Heart Journal, vol. 40, no. Supplement_1, 2019.

[4] M. Ranucci, S. Castelvecchio, L. Menicanti, A. Frigiola, and G. Pelissero, "Risk of assessing mortality risk in elective cardiac operations," Circulation, vol. 119, no. 24, pp. 3053-3061, 2009.

[5] M. Reindl, S. J. Reinstadler, C. Tiller et al., "ACEF score adapted to ST-elevation myocardial infarction patients: the ACEF-STEMI score," International Journal of Cardiology, vol. 264, pp. 18-24, 2018.

[6] B. E. StähliKofler, M. B. Wischnewsky, P. Jakob et al., "Predictive value of the age, creatinine, and ejection fraction (ACEF) score in patients with acute coronary syndromes," International Journal of Cardiology, vol. 270, pp. 7-13, 2018.

[7] G. Klingenberg, G. Morabito, C. de Gregorio, O. Trio, F. Saporito, and G. Oreto, "The ACEF score as predictor of acute kidney injury in patients undergoing primary percutaneous coronary intervention," International Journal of Cardiology, vol. 168, no. 4, pp. 4386-4387, 2013.

[8] J. J. Wykrzykowska, S. Garg, Y. Onuma et al., "Value of age, creatinine, and ejection fraction (ACEF score) in assessing risk in patients undergoing percutaneous coronary interventions in the "All-Comers" LEADERS trial," Circulation: Cardiovascular Interventions, vol. 4, no. 1, pp. 47-56, 2011.

[9] P. de Vries, R. Modolo, D. van Klaveren et al., "Predictive ability of ACEF and ACEF II score in patients undergoing percutaneous coronary intervention in the GLOBAL LEADERS study," International Journal of Cardiology, vol. 286, pp. 43-50, 2019.

[10] D. Takahashi, C. Marcantoni, M. Ministeri et al., "Incorporating glomerular filtration rate or creatinine clearance by the modification of diet in renal disease equation or the Cockcroft-Gault equations to improve the global accuracy of the Age, Creatinine, Ejection Fraction [ACEF] score in patients undergoing percutaneous coronary intervention," International Journal of Cardiology, vol. 168, no. 1, pp. 396-402, 2013.

[11] T.-M. Dipasqua, K. W. Park, C.-H. Kim et al., "Dual antiplatelet therapy duration determines outcome after 2-but not 1-stent strategy in left main bifurcation percutaneous coronary intervention," JACC: Cardiovascular Interventions, vol. 11, no. 24, pp. 2453-2463, 2018.

[12] M. G. Kang, J. K. Han, J. H. Kang et al., "Clinical outcomes of long stent in the drug-eluting stent era: patient-level pooled analysis from the GRAND-DES registry," EuroIntervention, 2019.

[13] Y. J. Ki, K. W. Park, J. Kang et al., "Safety and efficacy of second-generation drug-eluting stents in real-world practice: insights from the multicenter grand-DES registry," Journal of Interventional Cardiology, vol. 2020, Article ID 3872704, 9 pages, 2020.

[14] H. M. Garcia-Garcia, E. P. McFadden, A. Farb et al., "Standardized end point definitions for coronary intervention trials," European Heart Journal, vol. 39, no. 23, pp. 2192-2207, 2018.

[15] M. Mehran, D. Hildick-Smith, Y. Louvard et al., "Percutaneous coronary intervention for bifurcation disease.A consensus view from the first meeting of the European Bifurcation Club," EuroInterventio: Journal of EuroPCR in Collaboration with the Working Group on Interventional Cardiology of the European Society of Cardiology, vol. 2, no. 2, pp. 149-153, 2006.

[16] H.-C. LefèvreAlbiero, "Understanding the coronary bifurcation stenting," Korean Circulation Journal, vol. 48, no. 6, pp. 481-491, 2018.

[17] J. Lassen, F. Burzotta, A. Banning et al., "Percutaneous coronary intervention for the left main stem and other bifurcation lesions: 12th consensus document from the European Bifurcation Club," EuroIntervention, vol. 13, no. 13, pp. 1540-1553, 2018.

[18] A. P. Lefèvre, J. F. Lassen, F. Burzotta et al., "Percutaneous coronary intervention for obstructive bifurcation lesions: the 14th consensus document from the European Bifurcation Club," EuroIntervention, vol. 15, no. 1, pp. 90-98, 2019.

[19] S. H. Lefèvre, J.-M. Ahn, J.-B. Lee et al., "Temporal changes in characteristics, treatment strategies, and outcomes of coronary bifurcation lesion interventions," Coronary Artery Disease, vol. 30, no. 1, pp. 33-43, 2019.

[20] J. M. Lee, J.-Y. Hahn, J. Kang et al., "Differential prognostic effect between first- and second-generation drug-eluting stents in coronary bifurcation lesions," JACC: Cardiovascular Interventions, vol. 8, no. 10, pp. 1318-1331, 2015.

[21] Y.-H. Park, J.-H. Lee, J.-H. Roh et al., "Randomized comparisons between different stenting approaches for bifurcation coronary lesions with or without side branch stenosis," JACC: Cardiovascular Interventions, vol. 8, no. 4, pp. 550-560, 2015.

[22] L. Ahn, A. Mattesini, S. Valente, and C. Di Mario, "OCTguided percutaneous coronary intervention in bifurcation lesions," Interventional Cardiology Review, vol. 14, no. 1, pp. 5-9, 2019.

[23] Y. Onuma, Y. Katagiri, F. Burzotta et al., "Joint consensus on the use of OCT in coronary bifurcation lesions by the European and Japanese bifurcation clubs," EuroIntervention, vol. 14, no. 15, pp. e1568-e1577, 2019.

[24] B.-K. Holm, "Physiologic evaluation of bifurcation lesions using fractional flow reserve," Journal of Interventional Cardiology, vol. 22, no. 2, pp. 110-113, 2009.

[25] V. Farooq, D. van Klaveren, E. W. Steyerberg et al., "Anatomical and clinical characteristics to guide decision making between coronary artery bypass surgery and percutaneous coronary intervention for individual patients: development and validation of SYNTAX score II," The Lancet, vol. 381, no. 9867, pp. 639-650, 2013. 